\title{
On an effective solution of the Riemann problem for the second-order improperly elliptic equation in the rectangle
}

\author{
Armenak O Babayan ${ }^{1}$ and Seyed Mohammadali Raeisian² ${ }^{*}$
}

\section{"Correspondence:}

s_ma_raissian@yahoo.com

2Library of Institute of Mathematics (2nd floor), National Academy of

Sciences of Armenia, Baghramyan 24b, Yerevan, Republic of Armenia Full list of author information is available at the end of the article

\begin{abstract}
In this paper we present the numerical method for the solution of the Riemann problem for the second-order improperly elliptic equation. First, we reduce this problem to boundary value problems for properly elliptic equations, and after that we solve these problems by the grid method.
\end{abstract}

MSC: $35 \mathrm{G} 45 ; 35 \mathrm{G} 15 ; 35 J 25 ; 35 J 57 ; 65 \mathrm{~N} 06 ; 65 \mathrm{~N} 20$

Keywords: improperly elliptic equation; boundary value problem; Riemann problem; Bitzadze equation; grid method

\section{Introduction}

Let $D$ be a rectangle $D=\{(x, y): a<x<b, c<y<d\}$ in a complex plane with boundary $\Gamma=\partial D$. We consider in $D$ the equation

$$
\sum_{k=0}^{n} A_{k} \frac{\partial^{n} u}{\partial x^{k} \partial y^{n-k}}(x, y)=0, \quad(x, y) \in D
$$

where $A_{k}$ are complex constants $\left(A_{0} \neq 0\right)$ such that the characteristic equation

$$
\sum_{k=0}^{n} A_{k} \lambda^{n-k}=0
$$

has no real roots (i.e., elliptic equation). Let the roots of (2) $\lambda_{k}$ with multiplicities $m_{k}$ satisfy the condition $\Im \lambda_{k}>0$ and the roots $\mu_{j}$ with multiplicities $l_{j}$ satisfy the condition $\Im \mu_{j}<0$. We suppose that $\sum_{k} m_{k}>\sum_{j} l_{k}$, so (1) is an improperly elliptic equation. It was shown in [1] that for the equation $u_{\bar{z} \bar{z}}=0$ (now known as a Bitzadze equation) the corresponding Dirichlet problem is not correct. It was shown later ([2-4]) that for arbitrary improperly elliptic equation (1) all of the classical boundary value problems are not correct (we say that the problem is correct if the corresponding homogeneous problem has a finite number of linearly independent solutions and the inhomogeneous problem is solvable if and only if the finite number of linearly independent conditions for the boundary functions are satisfied). Therefore another kind of boundary conditions must be introduced. In the works [5-8], different types of boundary conditions, whose number depends on the number of the roots of (2) with positive and negative imaginary parts, were introduced. In this

\section{Springer}

C) 2013 Babayan and Raeisian; licensee Springer. This is an Open Access article distributed under the terms of the Creative Commons Attribution License (http://creativecommons.org/licenses/by/2.0), which permits unrestricted use, distribution, and reproduction in any medium, provided the original work is properly cited. 
paper we consider in $D$ the Bitzadze equation

$$
\frac{\partial^{2} u}{\partial \bar{z}^{2}}(x, y) \equiv \frac{1}{4}\left(\frac{\partial}{\partial x}+i \frac{\partial}{\partial y}\right)^{2} u(x, y)=0, \quad(x, y) \in D
$$

We seek the unknown function $u \in C^{2}(D) \cap C^{(1, \alpha)}(D \cup \Gamma)$ in the class of two times continuously differentiable in $D$ functions which satisfy Hölder condition in $D \cup \Gamma$ with their first degree derivatives. We suppose that the function $u$ satisfies the Riemann-type boundary conditions

$$
\left.\Re u\right|_{\Gamma}=f(x, y),\left.\quad \Re \frac{\partial u}{\partial v}\right|_{\Gamma}=g(x, y), \quad(x, y) \in \Gamma .
$$

Here $f \in C^{(1, \alpha)}(\Gamma), g \in C^{(\alpha)}(\Gamma)$ are the given functions on the boundary, $\frac{\partial}{\partial \nu}$-differentiation with respect to inner normal to $\Gamma$. Problem (3), (4) is well posed [2] and has a solution for arbitrary boundary functions. The corresponding homogeneous problem (when $f \equiv g \equiv$ 0) has four linearly independent pure imaginary solutions

$$
u_{0}(x, y)=i, \quad u_{1}(x, y)=i x, \quad u_{2}(x, y)=i y, \quad u_{0}(x, y)=i\left(x^{2}+y^{2}\right) .
$$

Therefore, the realization of the grid method for the solution of problem (3)-(4) is connected with some difficulties. The grid method (or finite-difference method) is an effective and widely applicable method for the solution of boundary value problems for elliptic equations with real coefficients (see, for example, [9]). But even in this case, it is necessary to do some modifications for the solution of the Neumann problem, because in this case we must get around difficulties connected with non-uniqueness of the solution ([10], p. 62). Application of this method to complex elliptic equations was considered in [11], but only for properly elliptic equations (linear and non-linear). In this paper we suggest a scheme of the realization of the grid method for the solution of problem (3)-(4). If, denoting $u=P+i Q(P=\bar{P}, Q=\bar{Q})$, we consider equation (3) as a system of the real partial differential equations,

$$
P_{x x}-Q_{y y}-2 Q_{x y}=0, \quad Q_{x x}-Q_{y y}+2 P_{x y}=0,
$$

then straightforward finite-difference realization of this system

$$
\begin{aligned}
& P_{i+1}^{j}+P_{i-1}^{j}-P_{i}^{j+1}-P_{i}^{j-1}-2\left(Q_{i+1}^{j+1}-Q_{i+1}^{j+1}-Q_{i+1}^{j-1}+Q_{i-1}^{j-1}\right)=0, \\
& Q_{i+1}^{j}+Q_{i-1}^{j}-Q_{i}^{j+1}-Q_{i}^{j-1}+2\left(P_{i+1}^{j+1}-P_{i+1}^{j+1}-P_{i+1}^{j-1}+P_{i-1}^{j-1}\right)=0
\end{aligned}
$$

reduces to ill-conditioned linear system with zeroes on the main diagonal, so we introduce another variant of the solution.

\section{Description of the algorithm of solution}

The general solution of equation (3) may be represented in the form [2]:

$$
u(x, y)=\bar{z} \Phi(z)+\Psi(z), \quad z=x+i y,(x, y) \in D
$$


where $\Phi, \Psi$ are arbitrary analytic functions in $D$. Therefore, problem (3)-(4) reduces to determination of the analytic functions $\Phi, \Psi$ by the condition (4). For this goal, we mention that if the function $u$ is a solution of problem (3)-(4), then the complex conjugate function $\bar{u}$ satisfies the equalities

$$
\begin{aligned}
& \frac{\partial^{2} \bar{u}}{\partial z^{2}}(x, y)=0, \quad z=x+i y,(x, y) \in D, \\
& \left.\Re \bar{u}\right|_{\Gamma}=f(x, y),\left.\quad \Re \frac{\partial \bar{u}}{\partial v}\right|_{\Gamma}=g(x, y), \quad(x, y) \in \Gamma .
\end{aligned}
$$

Hence, taking into account identity $\Delta^{2} \equiv 16 \frac{\partial^{4}}{\partial z^{2} \partial \bar{z}^{2}}$, we get that the function $U \equiv \Re u$ is a solution of the Dirichlet problem for the biharmonic equation

$$
\begin{aligned}
& \Delta^{2} U(x, y)=0, \quad(x, y) \in D \\
& \left.U\right|_{\Gamma}=f(x, y),\left.\quad \frac{\partial U}{\partial v}\right|_{\Gamma}=g(x, y), \quad(x, y) \in \Gamma,
\end{aligned}
$$

which is uniquely solvable $[6,7]$. Solving this problem by the grid method, we find approximate values of the function $U$ in the grid nodes:

$$
U_{i}^{j} \approx U\left(x_{i}, y_{j}\right), \quad i=1, \ldots, n ; j=1, \ldots, m
$$

Now we must find $\Im u$. From (6) we have

$$
U(x, y)=0.5(\bar{z} \Phi(z)+\Psi(z)+z \overline{\Phi(z)}+\overline{\Psi(z)}), \quad z=x+i y
$$

So, applying the Laplace operator to both sides of (11), we get

$$
\Delta U(x, y)=2\left(\Phi^{\prime}(z)+\overline{\Phi^{\prime}(z)}\right)=4 \Re\left(\Phi^{\prime}(z)\right)
$$

or

$$
\frac{\partial}{\partial x} \Re \Phi(z)=0.25 \Delta U(x, y)
$$

The function $\varphi(z) \equiv \Re \Phi(z)$ is harmonic in $D$; therefore, adding equalities (12) on the boundary of $D$, we get the following problem with angled derivative for the Laplace equation:

$$
\Delta \varphi=0, \quad(x, y) \in D ;\left.\quad \frac{\partial \varphi}{\partial x}\right|_{\Gamma}=\varphi_{0}(x, y) \equiv 0.25 \Delta U(x, y), \quad(x, y) \in \Gamma .
$$

Solving this problem, we get the function $\varphi$ - the real part of $\Phi$. To determine the imaginary part of $\Phi$, the function $\omega=\Im \Im$, we have the similar boundary value problem

$$
\Delta \omega=0, \quad(x, y) \in D ;\left.\quad \frac{\partial \omega}{\partial y}\right|_{\Gamma}=\omega_{0}(x, y) \equiv 0.25 \Delta U(x, y), \quad(x, y) \in \Gamma .
$$

Here (12) and the Cauchy-Riemann condition $\varphi_{x}=\omega_{y}$ were used. Solving the problems (13) and (14), we find the function $\Phi$. Now, we use (11) to determine the function $\Psi$. This 
function is also analytic in $D$; therefore, we have the Dirichlet problem for the Laplace equation to determine the real part of this function $\psi=\Re \Psi$ :

$$
\Delta \psi=0, \quad(x, y) \in D,\left.\quad \psi\right|_{\Gamma}=\psi_{0}(x, y), \quad(x, y) \in \Gamma .
$$

Here a function $\psi_{0}$ is the already known function $\psi_{0}=U-\Re(\bar{z} \Phi(z))$. After determining the function $\psi$, we get the boundary value problem, analogous to (13), to determine the imaginary part of the function $\Psi$.

Thus, the main idea of this algorithm is to reduce the previous problem for an improperly elliptic equation to the boundary value problems for properly elliptic equations (in our case, to boundary value problems for biharmonic and Laplace equations). In the next section we describe the realization of this algorithm by the grid method.

\section{Solution of problem (3), (4) by the grid method}

Without loss of generality, we may suppose that $a, b, c, d$ are rational numbers. In this case we can divide the rectangle $D$ by $(N-1)(M-1)$ equidistant straight lines, parallel to coordinate axes and denote

$$
x_{k}=a+k h, \quad y_{j}=c+j h, \quad h=\frac{b-a}{N}=\frac{d-c}{M}, k=\overline{0, N}, j=\overline{0, M} .
$$

We want to find the approximate values of unknown function $\left\{u\left(x_{k}, y_{j}\right)\right\}_{k, j=0}^{N, M}(u$ is a solution of problem (3), (4)) in the mesh points $\left(x_{k}, y_{j}\right)$. First, we consider problem (9) to determine the function $U(x, y)=\Re u(x, y)$. Passing to the discrete analogue of the Laplace operator

$$
\Delta_{h} U_{h}=\frac{U_{k+1}^{j}-2 U_{k}^{j}+U_{k-1}^{j}+U_{k}^{j+1}-2 U_{k}^{j}+U_{k}^{j-1}}{h^{2}}
$$

where $U_{h}=\left\{U_{k}^{j}\right\}$ is a grid function, we replace problem (9) by grid equations

$$
\Delta_{h} \Delta_{h} U_{h}=0,\left.\quad U_{h}\right|_{\Gamma_{h}}=f_{h},\left.\quad \delta_{h} U_{h}\right|_{\Gamma_{h}}=g_{h} .
$$

Here

$$
\begin{aligned}
\Delta_{h} \Delta_{h} U_{h}= & \frac{20}{h^{4}} U_{i}^{j}-\frac{8}{h^{4}}\left(U_{i+1}^{j}+U_{i-1}^{j}+U_{i}^{j-1}+U_{i}^{j+1}\right) \\
& +\frac{2}{h^{4}}\left(U_{i+1}^{j+1}+U_{i-1}^{j+1}+U_{i+1}^{j-1}+U_{i-1}^{j-1}\right)+\frac{1}{h^{4}}\left(U_{i+2}^{j}+U_{i-2}^{j}+U_{i}^{j-2}+U_{i}^{j+2}\right),
\end{aligned}
$$

and $\delta_{h}$ is a standard grid analogue (forward divided difference) of the operator $\frac{\partial}{\partial \nu}, f_{h}$ and $g_{h}$ are values of the functions $f$ and $g$ in boundary points of the grid. This problem approximates problem (9), and the rate of approximation is $O\left(h^{2}\right)$ for a biharmonic equation and $O(h)$ for boundary conditions (see [13]). Therefore, from the stability of problem (18), we get the convergence of the grid function to $\left\{U\left(x_{k}, y_{m}\right)\right\}$ ([9], p.30, Theorem 2.5). From the last two equations of (18), we get the values of the function $U_{h}$ in the points $\left(x_{k}, y_{m}\right)$ for $k=0,1, N-1, N ; m=0,1, \ldots, M$ and $k=0,1, \ldots, N ; m=0,1, M-1, M$, and we find the values $U_{k}^{m}$ in interior nodes from the linear system with a symmetric pentadiagonal matrix. Hence, we get the stability of (18) from the positive definiteness of the main matrix of this system, and an algorithm for the solution of this system may be found in [14]. 
Now, let us consider problem (13). First, replacing the Laplace operator by the grid operator (17), we find the values $0.25 \Delta_{h} U_{h}$ in the points $\Gamma_{1 h}=\left\{\left(x_{k}, y_{j}\right): k=1, N-1, j=\right.$ $\overline{1, M-1}$ or $k=\overline{1, N-1}, j=1, M-1\}$. It will be values of the grid function $\varphi_{0}$ in the boundary condition (13). Problem (13) is always solvable and the corresponding homogeneous problem has two linearly independent solutions. Therefore, if we get two arbitrary constants $L_{0}$ and $L_{1}$, and get

$$
\varphi(a+h, d-\tau)=L_{0}, \quad \varphi(b-h, c+\tau)=L_{1},
$$

then from the grid boundary conditions

$$
\frac{\varphi_{k+1}^{l}-\varphi_{k}^{l}}{h}=0.25\left(\Delta_{h} U_{h}\right)_{k}^{l}, \quad l=1, M-1 ; k=1, \ldots, N-1,
$$

we find values of the grid function $\varphi_{k}^{m}$ for $m=1, N-1$ on the sides of $\Gamma_{1 h}$ parallel to $O X$ axis. Values of $\varphi_{k}^{m}$ inside $\Gamma_{1 h}$ and on the sides $\Gamma_{1 h}$ parallel to $O Y$ axis will be found from the system of linear equations. The main matrix of this system may be reduced to the block tridiagonal form

$$
\Omega=\left(\begin{array}{cccc}
A & B & \ldots & 0 \\
B & A & \ldots & 0 \\
\ldots & \ldots & \ldots & \ldots \\
0 & 0 & \ldots & A
\end{array}\right)
$$

where

$$
\begin{aligned}
A & =\frac{1}{h^{2}}\left(\begin{array}{ccccccc}
-1 & 1 & 0 & 0 & \ldots & 0 & 0 \\
1 & -4 & 1 & 0 & \ldots & 0 & 0 \\
0 & 1 & -4 & 1 & \ldots & 0 & 0 \\
\ldots & \ldots & \ldots & \ldots & \ldots & \ldots & \ldots \\
0 & 0 & 0 & 0 & \ldots & -4 & 1 \\
0 & 0 & 0 & 0 & \ldots & 1 & -1
\end{array}\right), \\
B & =\frac{1}{h^{2}}\left(\begin{array}{ccccc}
0 & 0 & \ldots & 0 & 0 \\
0 & 1 & \ldots & 0 & 0 \\
\ldots & \ldots & \ldots & \ldots & \ldots \\
0 & 0 & \ldots & 1 & 0 \\
0 & 0 & \ldots & 0 & 0
\end{array}\right) .
\end{aligned}
$$

To this matrix, the maximum principle may be applied [12]; therefore this system is uniquely solvable. Then we must solve problem (14). We get the problem analogous to (13), and here we must get one more real constant $L_{2}$. After solution of this problem, we get the function $\Phi$ in the form

$$
\Phi(z)=\Phi_{0}(z)+i L_{0} z+\left(L_{1}+i L_{2}\right)
$$

in the grid points, here $L_{0}, L_{1}, L_{2}$ are the real constants. And at the final step, we must find the function $\Psi$. First, we solve the Dirichlet problem for the Laplace equation to determine 
the real part of $\Psi$, and then we determine the imaginary part of $\Psi$ solving the problem analogous to (13). Here we get the last arbitrary constant $L_{3}$.

Summing up, we can say that the previous problem may be reduced to boundary value problems for properly elliptic equations.

Competing interests

The authors declare that they have no competing interests.

\section{Authors' contributions}

Both authors prepared all parts of the paper together. Both authors read and approved the final manuscript.

\section{Author details}

${ }^{1}$ Moscovian str. 28, app. 39, Yerevan, 0002, Republic of Armenia. ${ }^{2}$ Library of Institute of Mathematics (2nd floor), National Academy of Sciences of Armenia, Baghramyan 24b, Yerevan, Republic of Armenia.

Received: 12 September 2012 Accepted: 31 May 2013 Published: 28 June 2013

\section{References}

1. Bitzadze, AV: On a uniqueness of the Dirichlet problem for elliptic partial differential equations. Usp. Mat. Nauk 3(6(28)), 211-212 (1948) (in Russian)

2. Bitzadze, AV: Boundary Value Problems for Elliptic Equations of Second Order. Nauka, Moscow (1966) (in Russian), Engl. Transl. North-Holland, Amsterdam (1968)

3. Tovmasyan, NE: Non-Regular Differential Equations and Calculations of Electromagnetic Fields. World Scientific, Singapore (1998)

4. Wendland, WL: Elliptic Systems in the Plane. Pitman, London (1979)

5. Bikchantaev, JA: The boundary value problem for the homogeneous elliptic equation with constant coefficients. Izv. Vysš. Učebn. Zaved., Mat. 6(157), 56-61 (1975) (in Russian)

6. Begehr, H: Boundary value problems in complex analysis. I. Bol. Asoc. Mat. Venez. 12(1), 65-85 (2005)

7. Begehr, H: Boundary value problems in complex analysis. II. Bol. Asoc. Mat. Venez. 12(2), 217-250 (2005)

8. Soldatov, AP: The method of theory of functions for the boundary value problems on the plane 1. Smooth case. Izv. Akad. Nauk SSSR, Ser. Mat. 55(5), 1070-1100 (1991) (in Russian)

9. Grossmann, C, Roos, H-G, Stynes, M: Numerical Treatment of Partial Differential Equations. Springer, Berlin (2007)

10. Marchuk, Gl: Methods of Computational Mathematics. Nauka, Moscow (1989) (in Russian)

11. Wen, GC: Approximate Methods and Numerical Analysis for Elliptic Complex Equations. Gordon and Breach, Amsterdam (1999)

12. Samarskij, AA: Theory of Difference Schemes. Dekker, New York (2001)

13. Collatz, L: The Numerical Treatment of Differential Equations. Springer, Berlin (1966)

14. Koulaei, MH, Toutounian, F: Factored sparse approximate inverse of block tridiagonal and block pentadiagonal matrices. Appl. Math. Comput. 184, 223-234 (2007)

Cite this article as: Babayan and Raeisian: On an effective solution of the Riemann problem for the second-order improperly elliptic equation in the rectangle. Advances in Difference Equations 2013 2013:190.

\section{Submit your manuscript to a SpringerOpen ${ }^{\odot}$ journal and benefit from:}

- Convenient online submission

Rigorous peer review

- Immediate publication on acceptance

Open access: articles freely available online

- High visibility within the field

- Retaining the copyright to your article 